\title{
3-4-year-old children rapidly adapt their predictions and use them to learn novel word meanings
}

Naomi Havron, Alex de Carvalho, Anne-Caroline Fiévet and Anne Christophe

École normale supérieure, ENS, EHESS, PSL University, LSCP, Département d'études cognitives, Paris, FR.

Manuscript accepted in Child Development

Adults create and update predictions about what speakers will say next. The current study asks whether prediction can drive language acquisition, by testing whether 3-4-year-old children $(n=45)$ adapt to recent information when learning novel words. The study used a syntactic context which can precede both nouns and verbs to manipulate children's predictions about what syntactic category will follow. Children for whom the syntactic context predicted verbs were more likely to infer that a novel word appearing in this context referred to an action, than children for whom it predicted nouns. This suggests that children make rapid changes to their predictions, and use this information to learn novel information, supporting the role of prediction in language acquisition.

Corresponding author information: Naomi Havron, 29 rue d'Ulm, Pavillon Jardin, rez-de-chaussée 75005 Paris, France

Phone: +33783888548, Fax: +33144322630, email: naomi.havron@mail.huji.ac.il 
Prediction has been proposed to be a fundamental aspect of cognition, supporting multiple levels of cognitive processing and learning (Clark, 2013). In language processing, for example, there is evidence that adults form predictions as to the likelihood of different syntactic structures, and update these predictions whenever they encounter a structure that does not match their expectations. Adults' initial expectations are based on a lifetime of linguistic experience, but as researchers manipulate exposure to structures in the input, the expectation to encounter these structures increases or decreases rapidly (Fine, Jaeger, Farmer, \& Qian, 2013; Kaschak, 2006, though see also Harrington Stack, James \& Watson, in press). Adults might have developed the ability to flexibly change their predictions through their lifelong expertise with language, as suggested by studies showing an increase in prediction in older ages (e.g., Huettig \& Janse, 2012) and with literacy (Mishra et al., 2012). However, it may also be that adults' ability to adapt to the current linguistic environment is a consequence of language learning occurring through prediction and error-minimization. In this vein, several researchers have proposed that prediction is important for language acquisition (e.g., Chang, Dell, \& Bock, 2006; Gambi, Pickering, \& Rabagliati, 2016; Sari Ylinen, Bosseler, Junttila, \& Huotilainen, 2017). To explore this question, we examine whether 3-4 year-old children draw on recently encountered information to revise their predictions about the likely syntactic category of upcoming words, and exploit these revised predictions to infer novel words' meanings.

We draw on two lines of research: Findings from the priming literature suggesting that children revise their syntactic expectations both in production and comprehension (e.g., Peter, Chang, Pine, Blything, \& Rowland, 2015; Thothathiri \& Snedeker, 2008); and evidence that infants can use syntactic context to infer the meaning of novel words (e.g., Huang \& Arnold, 2016; Oshima-Takane, Ariyama, Kobayashi, Katerelos \& Poulin-dubois, 2011; Waxman, Lidz, Braun, \& Lavin, 2009; Bernal, Lidz, Millotte, \& Christophe, 2007).

Research on syntactic priming in children - that is, their tendency to reuse recently encountered structures and process them faster - provides evidence that children learn from the distribution of structures in their input. Branigan and Messenger (2016) found that children's production of passives increased after priming, and increased even more in a second session held a week later. This suggests that priming is not just a result of activation of syntactic representations, but a manifestation of learning through adjusting expectations to the distribution of structures in the input. Recent studies also found that the more surprising or infrequent a prime is, the larger the priming effect children show - as predicted by error-based learning models of priming (e.g., Peter et al., 2015). Nonetheless, these findings do not speak to the crucial question of whether children's revised expectations are used when encountering unfamiliar input, such as novel words. To test whether children use their adapted expectations to learn, we focus on children's prediction of a novel word's syntactic category from its linguistic context. 
Children create expectations about the syntactic category of a following word, by relying on the distribution of structures in the input. For example, articles are usually followed by nouns (which tend to denote objects or agents), and pronouns are usually followed by verbs (which tend to denote actions). Infants as young as 18-months can already use these contexts to infer the possible meaning of novel words. When listening to a sentence like "It's a dax", they infer that "dax" refers to an object. Likewise, when listening to a sentence like "It's daxing", infants infer that "dax" refers to an action (He \& Lidz, 2017). This syntactic bootstrapping may account for children's ability to rapidly learn the meaning of novel words (Gleitman, 1990).

Although children are able to exploit context to build expectations about what will come next, there is little direct evidence that children update expectations rapidly enough to affect learning, and most importantly, no evidence that these updated expectations can guide the learning of novel linguistic information (Rabagliati, Gambi, \& Pickering, 2015). In the current study we ask whether exposure to a small amount of input that children can successfully process, triggers changes to their predictions about syntactic categories, when confronted with novel words. We manipulate the distribution of familiar nouns and verbs, within a syntactic frame which can contain either a noun or a verb. We present one group of children with this frame preceding familiar nouns, and the other with this same frame preceding familiar verbs. We expect that children will use this distributional information to infer the syntactic category of a novel word appearing in this context - expecting this novel word to be a noun if they have heard familiar nouns in this context, and to be a verb it they have heard familiar verbs in this context. If they do, this would be the first evidence that children's expectations are updated rapidly enough to allow prediction-based learning, and most importantly, that these adjusted expectations can guide language acquisition. This would simultaneously support a unified account of language processing and language learning, and shed light on the mystery of the speed and accuracy of children's language acquisition.

\section{METHOD}

All data and materials have been made publicly available via the Open Science Framework and can be accessed at https://osf.io/zzd9y/. The design and analysis plans were preregistered at the Open Science Framework and can also be accessed with the same link.

\section{Participants}

Our final sample consisted of 45 monolingual French-speaking children between the ages of 39.1 and 50.6 months ( 23 in the verb condition, 13 boys and 10 girls, mean age 45.3 months, $\mathrm{SD}=3.26 ; 22$ in the noun condition, 9 boys and 13 girls, mean age 45.4 months, $\mathrm{SD}=3.3$ ). They were recruited and tested in a middle- to high-socioeconomic status school in Paris, or in our babylab through direct contact with parents belonging to our database (these children can also be assumed to come from middle- to high-socioeconomic 
status homes). Out of 55 children originally tested, four were removed for technical difficulties, three for fussiness and crying, and one for naming the unfamiliar objects (e.g., saying "anteater" for a stuffed-animal we used in one video). We removed trials with more than 50\% missing data, as well as any child with more than two missing trials, resulting in the exclusion of two more children. Written parental consent was obtained for all children.

We also tested 47 adult university students, 23 in the verb-condition and 24 in the noun condition, as a control group. We report their results briefly, with the full data and results available on the OSF.

\section{Design}

In French, la petite can either be followed by a noun (e.g., la petite grenouille, "the little frog") or by a verb (e.g., la petite dort, "the little one is sleeping"). It is more likely to be followed by a noun: a search in the Paris child-directed-speech corpus (Morgenstern \& Sekali, 2009) found 720 instances where it was followed by a noun, and only 6 where it was followed by a verb. We manipulated the syntactic category that this context predicted by randomly assigning children to one of two conditions - a verb-condition and a noun-condition. In the induction phase, the verb-condition children were exposed to sentences in which la petite was predictive of verbs, that is, it was consistently followed by familiar verbs; while the nouncondition children were exposed to sentences in which it was predictive of nouns - consistently followed by familiar nouns. This phase was meant to create expectations for a different syntactic category in the different groups. In the test phase, we examined whether children utilize their adapted expectations to infer the meaning of novel words. If children adapted their expectations based on the few sentences they heard, then when hearing the same frame followed by a novel word (which could refer to either an action or an object), children from the verb-condition should be more likely to look at a girl performing a novel action, compared to children from the noun-condition; such a result would suggest they were more likely to categorize the novel word as a verb, on the basis of its preceding syntactic context.

\section{Materials and Procedure}

Participants were tested individually either in a quiet room in their school, or in our babylab in a soundattenuated double-walled booth. They were sitting facing a $27-\mathrm{in}$. screen positioned $70 \mathrm{~cm}$ away from them. Eye gaze was recorded by Eyelink-1000 placed below the screen (operating in remote mode). We used a 5-point calibration.

The induction phase included six trials - four induction trials and two fillers. On each of these trials, the participant saw two videos played side-by-side-on the screen: one showing a little girl performing an action, and another showing the same girl holding an object. We showed the same girl on both sides of the screen to prevent participants from choosing which one is la petite based on which child they judge to be 
smaller or younger. During the presentation of the two videos side-by-side, a women's voice (the last author) asked the child to look at one of the videos in a child-friendly fashion. For instance, for the pair of videos showing a girl holding a frog on one side, and a girl sleeping on the other side, participants assigned to the noun-condition would hear a sentence such as "Oh, regarde, la petite grenouille!" - "Oh look, the little frog!". In contrast, participants assigned to the verb-condition would hear "Oh, regarde, la petite dort!" "Oh look, the little one is sleeping!". For a sample trial see Fig. 1. The prosody was the same for noun and verb sentences. This was done by intentionally picking a prosody that is appropriate for both nouns or verbs, in which the three words belong to the same prosodic phrase; otherwise it would have been possible to disambiguate by using different prosodic structures (as shown in de Carvalho, Dautriche, Lin \& Christophe, 2017; de Carvalho, Dautriche, \& Christophe, 2016; see Fig. 2 for a graphical depiction of the prosodic contour, see a full acoustic analysis on the $\underline{\mathrm{OSF}})$. 
Fig. 1: Sample trial (target side is counterbalanced).

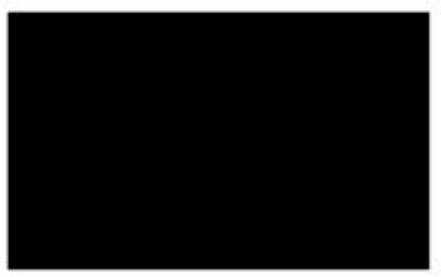

Oh, c'est quoi?

"oh what is that?"

( 8 seconds)
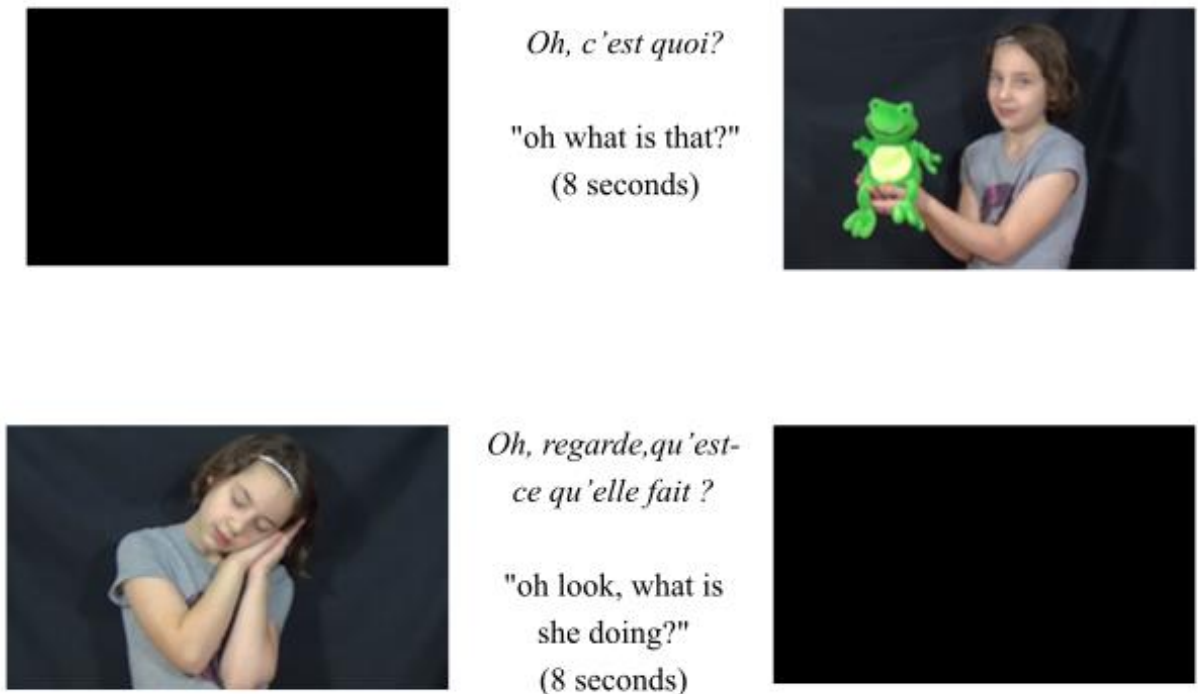

Oh, regarde,qu'estce qu'elle fait?

"oh look, what is she doing?"

( 8 seconds)
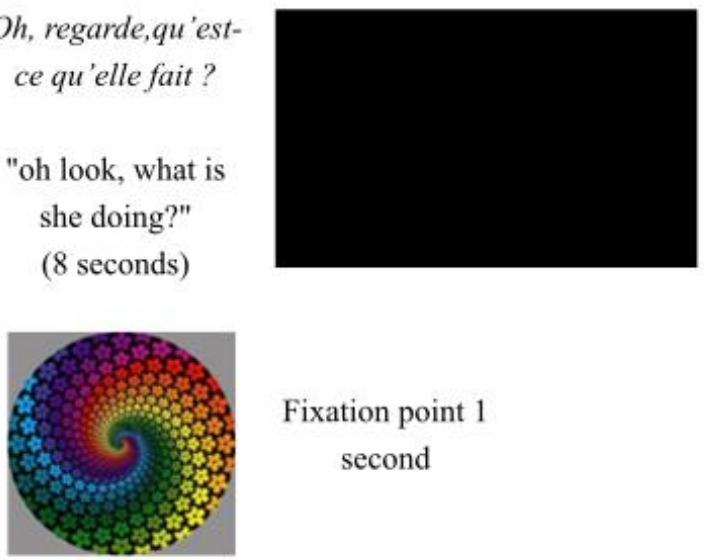

Fixation point 1 second

Oh, regarde, les deux ensemble!

"oh look, both together" (8 seconds)
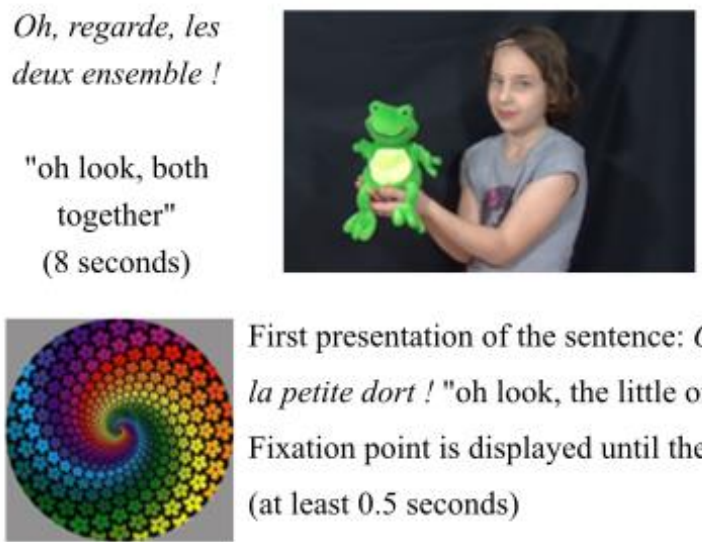

First presentation of the sentence: $O h$, regarde, la petite dort! "oh look, the little one is sleeping!" Fixation point is displayed until the gaze is on it (at least 0.5 seconds)

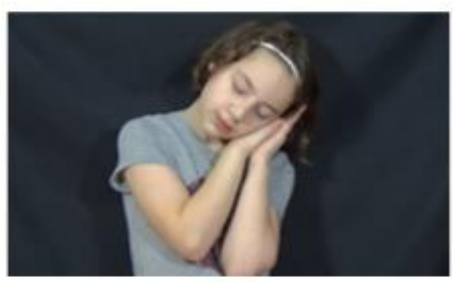

Oh, regarde, la petite dort! tu vois? la petite dort !

"oh look, the little one is sleeping! Do

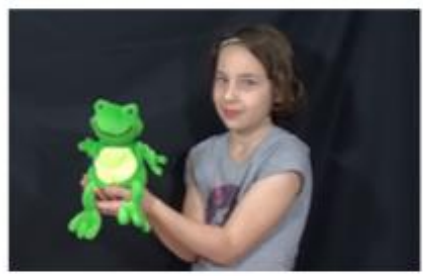
you see? The little one is sleeping!"

(10 seconds) 
Figure 2: Soundwave and pitch for one noun phrase (la petite balle, top), one verb phrase (la petite mange, middle), and one phrase with a novel word (la petite dase, bottom).

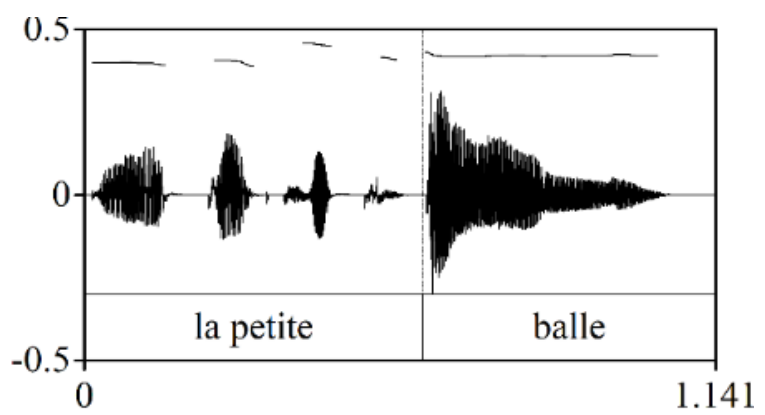

Time (s)

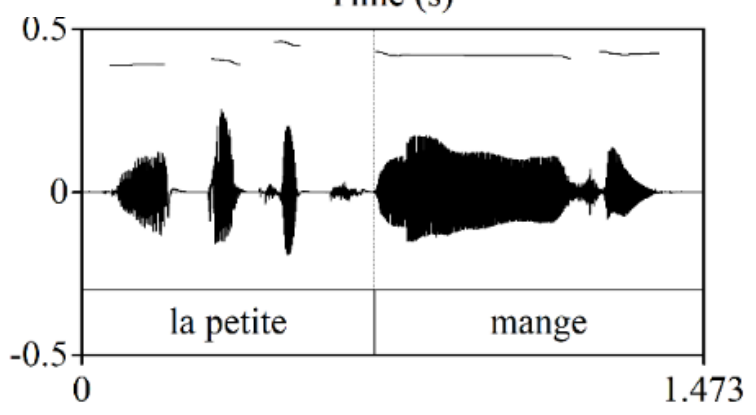

Time (s)

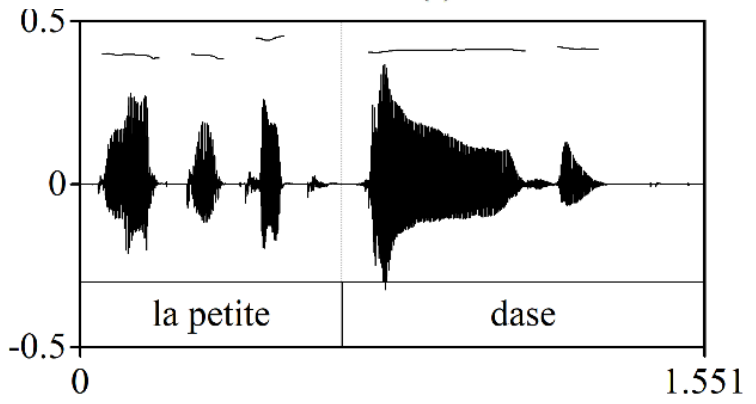

Time (s)

Two of the trials from the induction phase were filler items, which used an unambiguous syntactic structure (either c'est une Noun, "it's a Noun" or Elle Verb, "she's Verbing"'), and directed the children to look at the opposite video. For example, if a participant was in the verb-condition, they heard four la petite Verb sentences (e.g., la petite dort, "the little one is sleeping") and two C'est une Noun sentences (e.g., c'est une poussette, "this is a baby-stroller"). We used these two fillers so that participants are not always asked to look at the action (or the object in the noun-condition), but instead, realize that the woman can refer to either of the two videos. A difference between the two groups in the test phase should not therefore be attributed to them predicting that the woman in the experiment will always talk about the actions (or the objects), but to them predicting that the specific phrase la petite will be followed by either a verb or a noun. The order of the trials was randomized. 
In the test phase, consisting of three trials, participants still saw two videos, but this time the little girl in the videos was performing a novel action (which does not have a name in French) or holding an unfamiliar object (which does not have a name in French). While watching the videos play side by side, the participant heard Oh, regarde, la petite Novel-Word, "oh look, the little one is Novel-Wording" / "the little Novel-Word" (e.g., Oh, regarde, la petite nuve! "Oh look, the little one is nuving" / "the little nuve!"). The three test trials featured three different novel words (nuve, dase, and bamoule), each presented with one novel object and one novel action. Children in both conditions saw the same videos and heard the exact same sentences. The prosody of the test sentences was the same as that of the induction sentences ambiguous as to whether the final word is a noun or a verb. Note that even if there remain some prosodic cues that bias towards one interpretation (verb or noun), this does not affect the validity of the experiment, since both groups hear the exact same sentence, while watching the same videos. Therefore, the prosody cannot create any artefactual difference between groups. In order to ascertain that it is possible to interpret the novel word in the test sentences as referring either to a noun or a verb, we conducted an online survey, in which 26 adult native French speakers were asked to listen to the test sentences (without the visual context), and choose whether the novel word referred to an object, or to an action that a girl is performing. Participants judged the novel word to have referred to an action $31 \%$ of the time, confirming that the novel word could be interpreted as either a noun or a verb.

Each trial lasted less than a minute, with the full experiment lasting about 8 minutes.

All videos can be accessed on the OSF.

\section{Additional information}

In a fourth and final test-trial, participants watched a boy perform a novel action and the same boy holding a novel object, they were directed to look at le petit pirdale. Since participants were always exposed to la petite, and never to le petit (masculine version of la petite), we wanted to see whether what they have learned would generalize to le petit. This single trial is purely exploratory and we report the full results on the $\underline{\mathrm{OSF}}$.

\section{Statistical Analysis}

To test our hypothesis that children will use the information they were exposed to in the induction phase to infer the meaning of the novel words in the test phase, we conducted two statistical tests.

We first ran a cluster-based permutation analysis testing for a significant difference between conditions (as for example in Dautriche, Swingley, \& Christophe, 2015; see Maris \& Oostenveld, 2007, for a formal presentation of the analysis itself). We used R (R core team, 2017) and the package eyetrackingR (Dink \& Ferguson, 2016), and first down-sampled the data by averaging to one sample every 20 ms. For 
each time sample, the analysis runs a mixed-effects model testing for the effect of condition (on the arc-sin transformed proportion of looks toward the action video) with a random intercept for participant (we did not add a random intercept for item because there were only three items). Adjacent time-points with a tvalue greater than a predefined threshold $(\mathrm{t}=1.5)$ are grouped together into a cluster. This analysis was conducted on the entire trial ( 0 to 10 seconds from the beginning of the trial). Note that an effect may exist from the beginning of the trial since participants have heard the sentence played one time before the videos reappeared. Our preregistered analysis was a cluster-based permutation analysis with a t-test, since at the time of preregistration, we had no way to run a mixed-effects permutation analysis. Results for the t-test permutation analysis are similar, and available on the OSF.

Our second analysis was a mixed-effects regression (using the lme4 package, Bates, Maechler, Bolker, \& Walker, 2015) comparing the overall looking time at the action video, averaged across the whole trial, between the two conditions. The analysis included a random intercept for participant. We added the analysis of the overall looking time for two reasons: First, the cluster-based permutation analysis is a conservative test. Since we make no prior assumptions about when our effect will happen, we lose power when the effect is not highly situated, which can be expected in such an ambiguous context. Second, even strong advocates for this analysis recommend to supplement it by a more standard comparison of looking time (Delle, Durrant, Poltrock, \& Floccia, 2015).

\section{RESULTS}

The cluster-based analysis revealed a marginally significant cluster $(\mathrm{p}=0.054)$, between $3360 \mathrm{~ms}$ and $5100 \mathrm{~ms}$ after the beginning of the trial, coinciding with the first repetition of the target word during the test (see fig. 3): during that time-window, children from the verb-condition looked more at the action video than children from the noun-condition. 
Figure 3: Proportion of looks towards the action video in the test phase for the two conditions

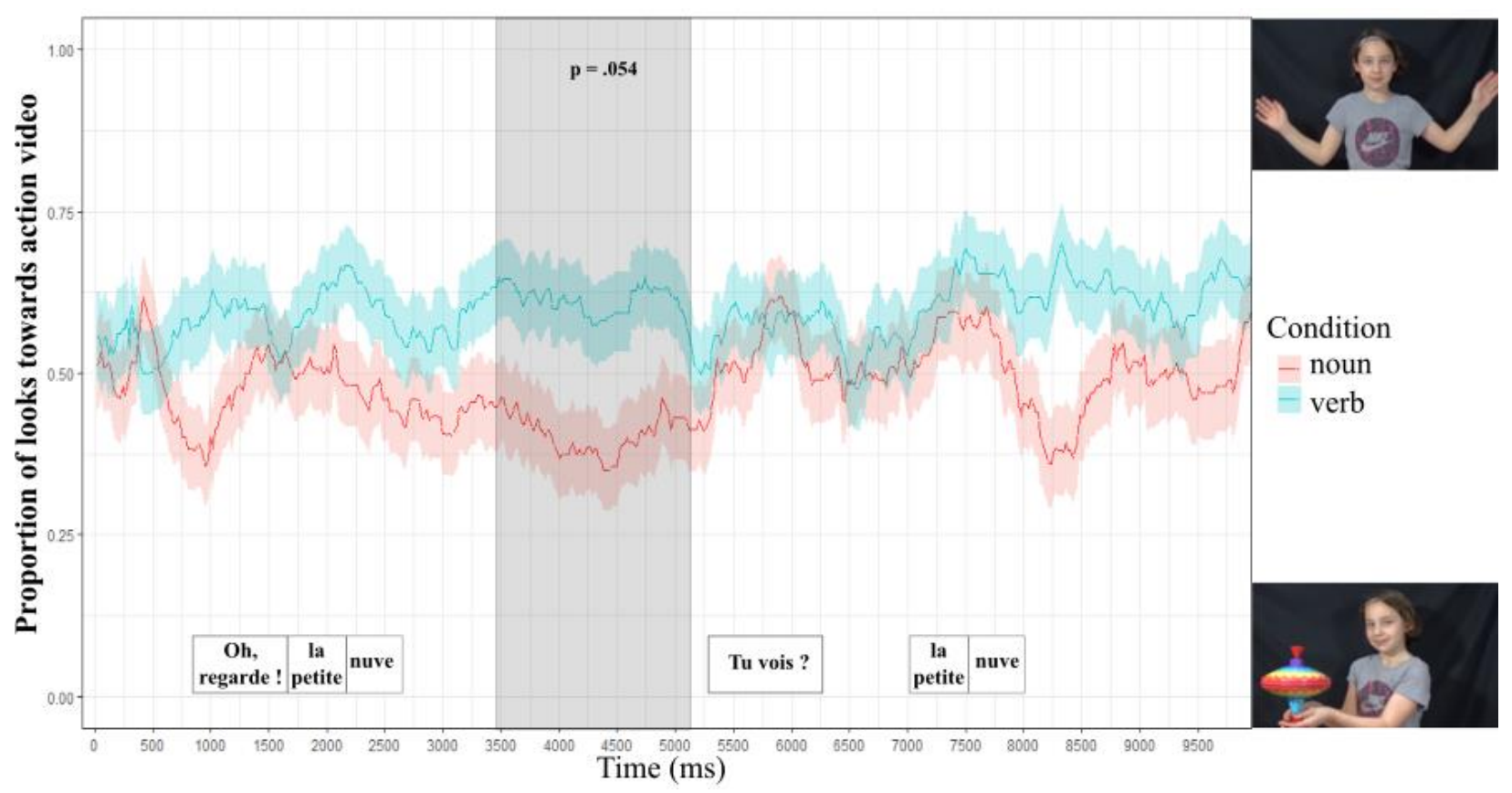

Proportion of looks towards the action-video, as a function of time, plotted for the entire duration of the trial (verbcondition in blue, noun-condition in red). The shaded area denotes the cluster which was marginally significant. Children from the verb-condition are consistently looking more at the action video than children from the noun condition (note that the target sentence was also heard once just before the beginning of the trial).

The mixed-effects regression conducted on the average overall looking time per participants found a large effect of condition: Children from the verb-condition $(M=0.602, S D=0.117)$, were significantly more likely to look at the action video than children from the noun-condition $(\mathrm{M}=0.482, \mathrm{SD}=0.139, \beta=.189$, $\mathrm{SE}=.06, \mathrm{t}=3.142, \mathrm{p}=.003$, Cohen's $\mathrm{d}=0.941)$, suggesting that children from the verb-condition were more likely to interpret the novel word as referring to the action than children in the noun-condition (see fig. 4). 
Figure 4: Mean overall proportion of looks towards the action video in the test phase for the two condition

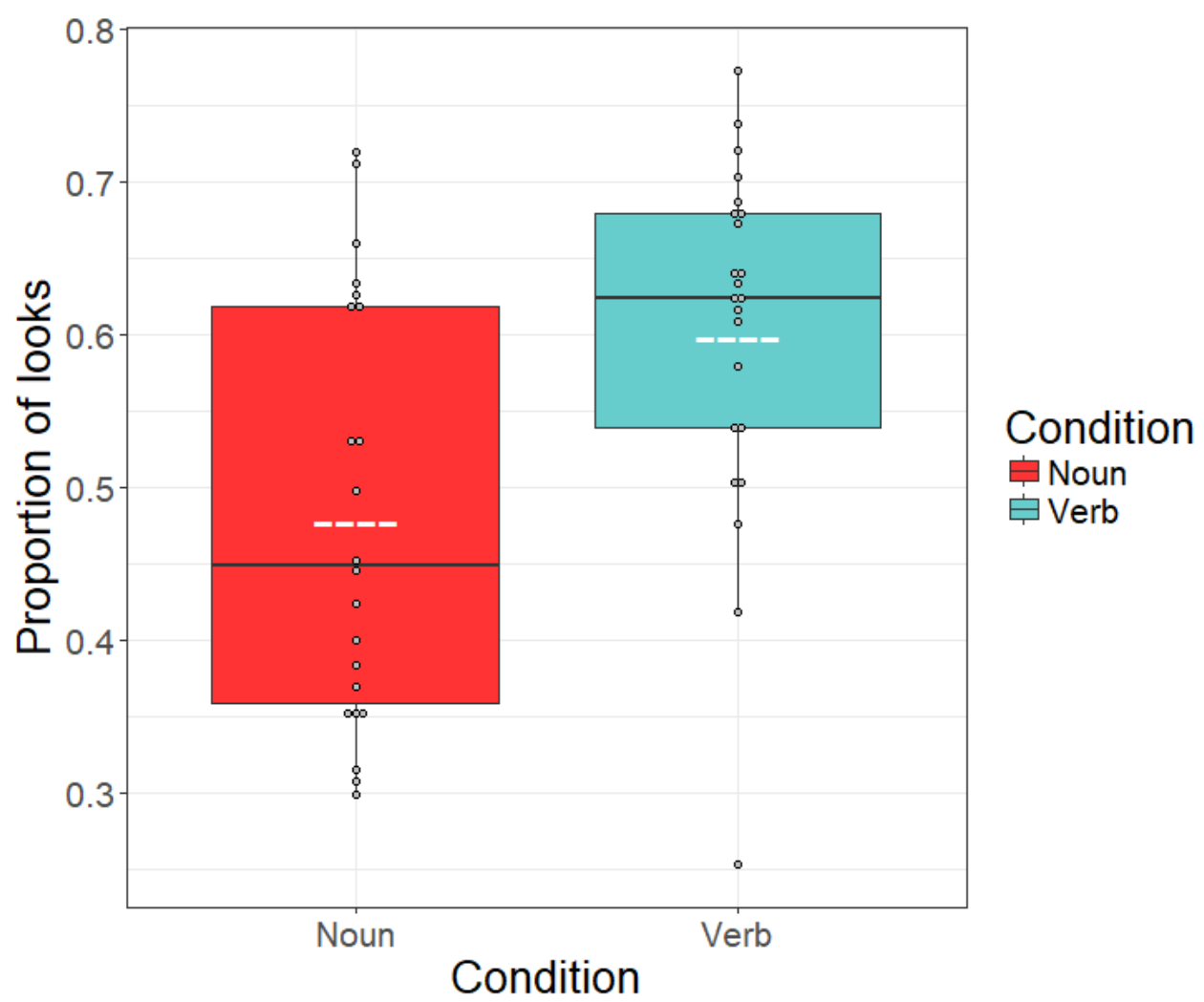

Proportion of looks to the action video, averaged over the entire duration of the trial (verb-condition in blue and noun-condition in red). Grey dots represent individual participants. The lower and upper hinges correspond to the first and third quartiles, the dotted white lines represent the means, and the black lines within the squares represent the median. The top whiskers denote the maximum value, and the bottom whiskers the minimum value (note that one participant in the verb group is an outlier - he was not removed from any of the analyses). Children from the verb-condition are looking at the action video significantly more than children from the noun-condition.

\section{Adult control group}

We found the same effect of condition in adults as we did in young children, except that the difference between conditions was larger for adults. The full results can be accessed on the OSF 


\section{DISCUSSION}

In the current study, we investigated whether children create predictions about the distribution of syntactic categories in a new environment, and draw on these to acquire word meanings. We found that children who heard examples of la petite + familiar verbs were more likely to infer that a novel word produced after la petite referred to an action, than children who heard la petite + familiar nouns sentences. This suggests that children did not only rapidly adapt their expectation to encounter a specific syntactic category (as was previously found in priming studies with children of this age, e.g., Thothathiri \& Snedeker, 2008), but also utilized their adapted predictions to learn. Importantly, our design included filler items within the induction phase, to exclude the possibility that children were biased to look at the action-video (or object-video) without having to take into account the specific sentences - it is thus unlikely that our effect is the result of a simple bias for either action or object-videos. This is the first study demonstrating that children adapt their predictions and use these adapted predictions to guide their learning of novel linguistic input.

One limitation of the present study is that since we do not have a control condition with no induction, it is possible that only children in one condition changed their predictions; for instance, children in the noun-condition might have looked at the novel object because it was the default choice of

interpretation, meaning only the verb-condition children changed their predictions. This would be in-line with previous studies, which found larger priming effects for the less-frequent structure (e.g., Kholodova, et. al, 2017).

Children showed different expectations after only four induction trials, which implies rapid adaptation and high sensitivity to structures in the input. This rapidity would be particulary useful since words seem to follow a "bursty" distribution in language (Altmann, Pierrehumbert, \& Motter, 2009). Parents may repeatedly use la petite+verb when talking about a doll or a little girl, but revert back to the more common la petite+noun when changing the subject. It would be interesting to test in the future whether children form predictions that are conditioned on the context or speaker, as has been found in adults (e.g., Kamide, 2012; Kroczek \& Gunter, 2017) - or whether they would also generalize to new speakers and contexts.

This experiment could be interpreted as showing that information provided to the languageprocessing system affects the language-learning system. However, in the sense that processing changes children's expectations, it produces the same kind of effect that we would normally call learning, and there is no sense in talking about two different systems. Language acquisition is in fact a process by which the child is learning to process language (Chang, Dell, \& Bock, 2006; Pickering \& Garrod, 2013). As the child hears la petite before familiar verbs, she processes these sentences to both comprehend them, and change the model she relies on for processing and learning novel linguistic materials. Thus, whether the next 
sentence contains a word she knows (processing) or she does not know (learning), she will rely on her adapted expectations, to predict to what category the word following la petite will belong.

Our findings show that prediction does not only facilitate, but also guides learning. This means that predictions do not only help children learn by reducing the cost associated with processing the input, thus clearing resources for learning - they also structure the space of possibilities for meaning. As to the exact mechanisms by which children compute and use their expectations, there are several possible accounts. Under the predictive-coding framework, the brain is thought of as a prediction machine, whose role is to capture the structure of input it encounters. The brain accomplishes this by predicting future input, and correcting its predictions when it encounters a prediction error. This is achieved through a hierarchical generative model that aims to minimize error within a bidirectional cascade of information (Clark, 2013; Sohoglu et al., 2012). Our results are in line with such a mechanism, but they are also in line with other mechanisms of learning through prediction. For example, children could be creating predictions using analogy (see Bar, 2015); or they could be updating their predictions by cumulating frequencies - not privileging evidence of error over correct predictions (for a discussion of the difference between this account and error-based predictions, see Corlett et al, 2004).

The importance of our findings does not apply only to ambiguous sentences containing novel words. They might also explain how children initially learn unambiguous contexts. According to the semantic seed hypothesis (Christophe, Dautriche, de Carvalho \& Brusini, 2016), infants could manage to learn the meaning of a handful of very frequent words (Bergelson \& Swingley, 2012, 2013), group them into objects and actions (Carey, 2009), and use these known words to learn to predict action-words after certain linguistic contexts, and object-words after others. They may then use these expectations to constrain the meaning of novel words (Gleitman, 1990; He \& Lidz, 2017). Our results illustrate how children rapidly form these predictions, and suggest a mechanism which could be useful not only in cases where the structure is ambiguous, but also for fully unambiguous syntactic contexts (such as articles and personal pronouns).

To conclude, it was previously suggested that children make rapid changes to their linguistic expectations following exposure to new linguistic evidence, and that they use this information when processing and learning new linguistic materials. We found that 3-4-year-old children indeed make rapid use of prediction at the syntactic level, and use newly-adjusted expectations to infer novel word meaning. Prediction may thus be a key component not only in language processing in expert language users, but in children's language acquisition.

\section{Acknowledgements}

This work was supported by a postdoctoral grant to the first author from the French Embassy in Israel and The Victor Smorgon Charitable Fund, and by the French Agence Nationale de la Recherche (grants $\mathrm{n}^{\circ}$ ANR-13-APPR-0012, ANR-10-IDEX-0001-02 PSL* and ANR-10-LABX-0087 IEC). We thank Axel 
Barrault for help with testing; Isabelle Dautriche for help with data analysis; the school and children for their participation, as well as the children who participated in the videos and their parents. 


\section{Bibliography}

Altmann, E. G., Pierrehumbert, J. B., \& Motter, A. E. (2009). Beyond word frequency: Bursts, lulls, and scaling in the temporal distributions of words. PLOS one, 4, e7678.

Bar, M. (2007). The proactive brain: using analogies and associations to generate predictions.

Trends in cognitive sciences, 11, 280-289.

Bates, D., Maechler, M., Bolker, B. \& Walker, S. (2015). Fitting Linear Mixed-Effects Models

Using lme4. Journal of Statistical Software, 67, 1-48. doi:10.18637/jss.v067.i01.

Bergelson, E., \& Swingley, D. (2013). The acquisition of abstract words by young infants. Cognition, 127, 391-397.

Bergelson, E., \& Swingley, D. (2012). At 6-9 months, human infants know the meanings of many common nouns. Proceedings of the National Academy of Sciences, 109, 3253-3258.

Bernal, S., Lidz, J., Millotte, S., \& Christophe, A. (2007). Syntax constrains the acquisition of verb meaning. Language Learning and Development, 3, 325-341.

Branigan, H. P., \& Messenger, K. (2016). Consistent and cumulative effects of syntactic experience in children's sentence production: Evidence for error-based implicit learning. Cognition, 157, 250-256.

Carey, S. (2009). The origin of concepts: Oxford University Press

Chang, F., Dell, G. S., \& Bock, K. (2006). Becoming syntactic. Psychological Review, 113, 234-272. https://doi.org/10.1037/0033-295X.113.2.234

Christiansen, M. H., \& Chater, N. (2016). The Now-or-Never Bottleneck : A Fundamental Constraint on Language. Behavioral and Brain Sciences, 39, 1-72.

Clark, A. (2013). Whatever next? Predictive brains, situated agents, and the future of cognitive science. The Behavioral and Brain Sciences, 36, 181-204. https://doi.org/10.1017/S0140525X12000477

Christophe, A., Dautriche, I., de Carvalho, A. \& Brusini, P. (2016) Bootstrapping the syntactic bootstrapper. In J. Scott \& D. Waughtal (Eds). Proceedings of the 40th Boston University Conference on Language Development. (pp. 75-88). Somerville, MA; Cascadilla Press.

Corlett, P. R., Aitken, M. R., Dickinson, A., Shanks, D. R., Honey, G. D., Honey, R. A., ... \& Fletcher, P. C. (2004). Prediction error during retrospective revaluation of causal associations in humans: fMRI evidence in favor of an associative model of learning. Neuron, 44, 877-888.

Dautriche, I., Swingley, D., \& Christophe, A. (2015). Learning novel phonological neighbors: Syntactic category matters. Cognition, 143, 77-86.

de Carvalho, A., Dautriche, I., \& Christophe, A. (2016). Preschoolers use phrasal prosody online to constrain syntactic analysis. Developmental science, 19, 235-250.

de Carvalho, A., Dautriche, I., Lin, I., \& Christophe, A. (2017). Phrasal prosody constrains syntactic analysis in toddlers. Cognition, 163, 67-79. 
Delle Luche, C., Durrant, S., Poltrock, S., \& Floccia, C. (2015). Infant Behavior and Development A methodological investigation of the Intermodal Preferential Looking paradigm : Methods of analyses , picture selection and data rejection criteria. Infant Behavior and Development, 40, 151-172. https://doi.org/10.1016/j.infbeh.2015.05.005

Dink, J. \& Ferguson, B. (2016)._eyetrackingR_. R package version 0.1.6, <URL: http://www.eyetracking-R.com>.

Farmer, T. A., Brown, M., \& Tanenhaus, M. K. (2013). Prediction, explanation, and the role of generative models in language processing. Behavioral and Brain Sciences, 36, 211-212. https://doi.org/10.1017/S0140525X12002312

Fine, A. B., Jaeger, T. F., Farmer, T. a, \& Qian, T. (2013). Rapid Expectation Adaptation during Syntactic Comprehension. PloS One, 8, e77661. https://doi.org/10.1371/journal.pone.0077661

Gambi, C., Pickering, M. J., \& Rabagliati, H. (2016). Beyond associations: Sensitivity to structure in preschoolers' linguistic predictions. Cognition, 157, 340-351.

Gleitman, L. (1990). The Structural Sources of Verb Meanings. Language Acquisition, 1, 3-55.

Harrington Stack, C.M. James, A.N. \& Watson, D.G. (in press). A failure to replicate rapid syntactic adaptation in comprehension, Memory and Cognition.

He, A. X., \& Lidz, J. (2017). Verb learning in 14-and 18-month-old English-learning infants. Language Learning and Development, 13, 335-356.

Huang, Y. T., \& Arnold, A. R. (2016). Word learning in linguistic context: Processing and memory effects. Cognition, 156, 71-87.

Huettig, F., \& Janse, E. (2012). Anticipatory eye movements are modulated by working memory capacity: Evidence from older adults. In the 18th Annual Conference on Architectures and Mechanisms for Language Processing.

Jaeger, T. F., \& Snider, N. E. (2013). Alignment as a consequence of expectation adaptation: Syntactic priming is affected by the prime's prediction error given both prior and recent experience.

Cognition, 127, 57-83. https://doi.org/10.1016/j.cognition.2012.10.013

Kaschak, M. P. (2006). What this construction needs is generalized. Memory \& Cognition, 34, 368-379. https://doi.org/10.3758/BF03193414

Kholodova, A., Peter, M., Rowland, C., \& Allen, S. (2017). Syntactic Priming in a Structurally Biased Language: The Role of Cross-Linguistic Differences in Language Learning, Talk presented at Many Paths to Language, Nijmegen, the Netherlands.

Maris, E., \& Oostenveld, R. (2007). Nonparametric statistical testing of EEG-and MEG-data. Journal of neuroscience methods, 164, 177-190. 
Mishra, R. K., Singh, N., Pandey, A., \& Huettig, F. (2012). Spoken language-mediated anticipatory eyemovements are modulated by reading ability-Evidence from Indian low and high literates. Journal of Eye Movement Research, 5, 1-10.

Morgenstern, A. \& Sekali, M. (2009) What can child language tell us about prepositions ? In Zlatev, J., Johansson Falck, M., Lundmark, C. \& Andrén, M. (Eds). Studies in Language and Cognition (pp. 261-275). Cambridge, UK: Cambridge Scholars Publishing

Oshima-Takane, Y., Ariyama, J., Kobayashi, T., Katerelos, M., \& Poulin-Dubois, D. (2011). Early verb learning in 20-month-old Japanese-speaking children. Journal of child language, 38, 455-484. doi.org/10.1017/S0305000910000127

Peter, M., Chang, F., Pine, J. M., Blything, R., \& Rowland, C. F. (2015). When and how do children develop knowledge of verb argument structure? Evidence from verb bias effects in a structural priming task. Journal of Memory and Language, 81, 1-15. https://doi.org/10.1016/j.jml.2014.12.002

Pickering, M. J., \& Garrod, S. (2013). An integrated theory of language production and comprehension. Behavioral and Brain Sciences, 36, 329-347. https://doi.org/10.1017/S0140525X12001495

R Core Team (2017). R: A language and environment for statistical computing. $R$ Foundation for Statistical Computing, Vienna, Austria. URL https://www.R-project.org/.

Rabagliati, H., Gambi, C., \& Pickering, M. J. (2016). Learning to predict or predicting to learn? Language, Cognition and Neuroscience, 31, 94-105. https://doi.org/10.1080/23273798.2015.1077979

Sohoglu, E., Peelle, J. E., Carlyon, R. P., \& Davis, M. H. (2012). Predictive top-down integration of prior knowledge during speech perception. Journal of Neuroscience, 32, 8443-8453. https://doi.org/10.1523/JNEUROSCI.5069-11.2012

Thothathiri, M., \& Snedeker, J. (2008). Give and take: Syntactic priming during spoken language comprehension. Cognition, 108, 51-68.

Waxman, S., Lidz, J., Braun, I. E., \& Lavin, T. (2009). 24-Month-Old Infants Interpretations of Novel Verbs and Nouns in Dynamic Scenes. Cognitive Psychology, 59, 67-95. https://doi.org/10.1016/j.cogpsych.2009.02.001.24-Month-Old

Ylinen, S., Bosseler, A., Junttila, K., \& Huotilainen, M. (2017). Predictive coding accelerates word recognition and learning in the early stages of language development. Developmental science, 20(6), e12472. https://doi.org/10.1111/desc.12472 